\title{
CD163 and CCR7 as markers for macrophage polarization in lung cancer microenvironment
}

\author{
IWONA KWIECIEN' ${ }^{1}$, MAEGORZATA POLUBIEC-KOWNACKA², DARIUSZ DZIEDZIC ${ }^{2}$, \\ DOMINIKA WOEOSZ 3 , PIOTR RZEPECKI ${ }^{4}$, JOANNA DOMAGAEA-KULAWIK
}

\author{
${ }^{1}$ Department of Internal Medicine and Hematology, Laboratory of Flow Cytometry, Military Institute of Medicine, Warsaw, Poland \\ ${ }^{2}$ Department of Surgery, Institute of Tuberculosis and Lung Diseases, Warsaw, Poland \\ ${ }^{3}$ Department of Pathology, Medical University of Warsaw, Warsaw, Poland \\ ${ }^{4}$ Department of Internal Medicine and Hematology, Military Institute of Medicine, Warsaw, Poland \\ ${ }^{5}$ Department of Internal Medicine, Pulmonary Diseases, and Allergy, Medical University of Warsaw, Warsaw, Poland
}

\begin{abstract}
Introduction: M2 macrophages are predominant in the immune infiltrates of resected tumours, but little is known about macrophage phenotype in the local lung cancer environment, which may be evaluated by bronchoalveolar lavage fluid (BALF).

Aim of the study: To find differences between BALF from lung affected by cancer (clBALF) and hlBALF from the opposite, healthy lung, as a control, from the same patient, regarding their individual macrophage polarization and their correlation with IL-10 and TGF- $\beta$.

Material and methods: Eighteen patients with confirmed lung cancer were investigated. Macrophage subtyping was performed by immunofluorescence with antibodies anti-CCR7 and CD163 (M1 and $M 2$, respectively).

Results: We found five populations of macrophages: cells with a single reaction: only for CCR7+ or CD163+, a double reaction $(C C R 7+C D 163+)$, cells with a stronger CD163 $\left(C C R 7_{\text {low }} C D 163+\right)$, and cells with a stronger $C C R 7\left(C C R 7+C D 163_{\text {low }}\right)$. The main population in the clBALF was composed of cells with a phenotype similar to $M 2\left(C C R 7_{\text {low }} C D 163+\right)$, while in the hlBALF the predominating phenotype was the one similar to $M 1\left(C C R 7+C D 163_{\text {low }}\right)$. The median proportion of TGF- $\beta 1$ concentration was higher in the ClBALF and hlBALF supernatant than in the serum.

Conclusions: In this study we confirmed the usefulness of the immunofluorescence method with $C C R 7$ and CD163 in the evaluation of BALF macrophage polarization in lung cancer.
\end{abstract}

Key words: M1 macrophages, M2 macrophages, bronchoalveolar lavage fluid, CCR7, TGF- $\beta 1$, lung cancer.

(Centr Eur J Immunol 2019; 44 (4): 395-402)

\section{Introduction}

Lung cancer is one of the main oncological problems worldwide. The prognosis is very poor, and it is the first cause of cancer deaths among patients with malignancy. However, recently the efficacy of new immunotherapy methods with checkpoint blockers has been demonstrated in about $40 \%$ of patients with non-small cell lung cancer (NSCLC) [1-3].

Macrophages may play a crucial role in the suppression of immune anticancer response, whereas the direction of macrophage polarization and the character of dominant subpopulation require an explanation in the location of the tumour growth. The pro- tumour effect of cytokines such as: IL-10 and TGF- $\beta 1$ in local vs. systemic immune response requires elucidation. The recognition of these suppres- sor-regulatory elements, which may be evaluated by bronchoalveolar lavage fluid (BALF) examination, may have some implications for further therapeutic decision making.

\section{Macrophages}

Macrophages form the largest population of cells in the BALF. Nowadays macrophages are recognised as a heterogeneous and dynamic population of cells that have the capacity to perform a wide range of critical functions $[4,5]$. There are two functional subsets: macrophages classically activated (M1), defined by antimicrobial and cytotoxic properties, and alternatively activated macrophages (M2), which present anti-inflammatory and regulatory properties. M1 have pro-inflammatory activity and produce high concentrations of IL-12, IL-23, TNF- $\alpha$, IL-1,

Correspondence: Iwona Kwiecień, PhD, Department of Internal Medicine and Hematology, Laboratory of Flow Cytometry, Military Institute of Medicine, 128 Szaserów St., 04-141 Warsaw, Poland, e-mail: kwiecieniwi@gmail.com Submitted: 16.01.2019; Accepted: 1.04.2019 
and IL-6 [6]. M2 macrophages secrete cytokines IL-1RA, IL-10, TGF- $\beta$, and VEGF [7]. Due to proangiogenic and immunosuppressive activity, M2 macrophages have been shown to promote the development of tumours [8, 9]. It has also been shown that M2 are capable of inducing differentiation of $\mathrm{T}$ regulatory cells (Tregs) [10] whereas Tregs, by secretion of IL-10, promote polarization in the M2 direction [11]. Accordingly to recently published guidelines, the nomenclature linked to the agent of macrophage activation is rational, i.e. M (IL-4), M (IL-10), etc. [12]. However, it is possible in cell culture, in vivo studies being rather difficult [12].

In addition to the various means of activation and secretion of diverse cytokines, a macrophage population can be identified by the presence of specific surface markers. It has been shown that CD163 and CD206 are markers characteristic for the M2 population [13, 14]. M1 macrophages are characterised by high expression of the MHC II molecule and CD80/CD86 coenzyme modulating molecules [15]. Another marker is CD40, which is involved in the proinflammatory pathways, and its surface expression on macrophages is increased after induction by INF- $\gamma$. However, the specificity of this marker for M1 was not established [16-18]. Another marker is chemokine receptor 7 (C-C chemokine receptor type 7, CCR7), also known as CD197, involved in controlling migration of memory cells in lymph nodes and the stimulation of dendritic cell (DC) maturation $[19,20]$. The expression of CCR7 receptor on macrophages was demonstrated [21] and may be a specific marker for M1 macrophages. The expression of CCR7-specific ligands: CCL19 and CCL21 was found to be present at the onset of inflammation [22, 23].

To distinguish between M1 and M2 by phenotype analysis is difficult; we observed high plasticity of these cells in previous studies [18, 24]. Macrophages have the ability to rapidly adapt to changes in the environment that may result in their function switching, as shown by the problems with proper characterisation of specific M1, M2 function. This high plasticity hampers their unequivocal identification. The presence of macrophages in the tumour microenvironment is associated with increased tumour progression, cancer cell proliferation, angiogenesis, and immunosuppression. On the other hand, macrophages are fundamental part of innate defence mechanisms that can promote a specific type of immunity by inducing T-lymphocyte recruitment and activation. This paradoxically opposed role of macrophages in cancer is connected with the high plasticity of macrophages. It seems that the main elements influencing the direction of polarization are the signals from the tumour microenvironment [7].

\section{TGF- $\beta 1$ and IL-10}

TGF- $\beta 1$ plays an important role in controlling the immune system and shows a wide variety of activities on many different types of cell. TGF- $\beta$ is an immunosup- pressive cytokine known to be overexpressed in tumours playing an important role in the inhibition of antitumour immune response and tumour progression [25]. Recent studies have revealed that TGF- $\beta$ can suppress or alter the activation, maturation, and differentiation of macrophages, DCs, and neutrophils [26]. Although the role of TGF- $\beta$ in T-cell immunity has been described, surprisingly, few studies have evaluated the effect of TGF- $\beta$ signalling on macrophages. TGF- $\beta$ in the tumour microenvironment may block M1 macrophage development, while promoting the alternative activation of M2 macrophages [27].

There are some data on the role of IL-10 in the regulation of immune reaction. IL-10 is an anti-inflammatory cytokine, which is able to limit inflammatory responses and promote the development of Tregs [28]. Moreover, the elevated concentration of IL-10 plays an important role in macrophage polarization [29].

\section{BALF}

The main data of the macrophage polarization in cancer are restricted to studies of resected tumours. However, the resection rate in NSCLC is as low as $20-30 \%$. Bronchoalveolar lavage (BAL) is a method of investigation of the lung and provides the evaluation of local immune response in the lung cancer environment [30-32]. BAL may be performed in lung cancer also in advanced disease stages during diagnosis [30]. This was one of the reasons for the choice of BAL fluid (BALF) analysis as the main material of our research.

The aim of the study was to find differences between healthy lung and lung affected by cancer regarding their individual macrophage polarization and their correlation with IL-10 and TGF- $\beta$. We investigated the proportion of macrophage subpopulations and TGF- $\beta 1$ and IL-10 concentration in these two compartments based on the examination of BALF from the lung affected by cancer (clBALF - local environment) and from the "healthy" lung (hlBALF - a control) from each patient, and we evaluated the relations between them. Moreover, the cytokine concentration was also measured in the serum, which characterises the changes in systemic environment.

\section{Material and methods}

\section{Patients}

The study group consisted of 18 patients with confirmed primary NSCLC. There were six women and 12 men; mean age: $68.4 \pm 7.3$ years; range (min-max): 50-81 years. There were patients in stages I-III of the disease (according to the seventh TNM classification) [33].

All patients underwent clinical examination: bronchoscopy with BALF (Department of Surgery, National Institute of Tuberculosis and Lung Diseases, Warsaw, Poland). Each patient provided written, informed consent before 
each diagnostic procedure. Primary lung cancer confirmed by histological examination constituted an inclusion criterion. The exclusion criteria were as follows: any type of prior or recent anti-cancer therapy, clinical signs of infection, recognised chronic obstructive pulmonary disease (COPD), autoimmune diseases, and immunosuppressive treatment. Further exclusion criteria were established following BALF macro-scale and microscopic examination. Bloody BALF, less than 50\% recovered fluid, non-representative number of cells or no macrophages in the smears.

\section{Bronchoalveolar lavage fluid}

Bronchoalveolar lavage was performed during a routine bronchofiberoscopy in the course of lung cancer diagnosis. $100 \mathrm{ml}$ of $0.9 \% \mathrm{NaCl}$ solution was instilled to each lung. BALF was taken from the cancerous lung (clBALF) and from the healthy lung (hlBALF) of the same patient during the same procedure. The volume of recovery fluid was $50 \%$ or more. BALF processing was performed according to the recommendations [34]. The material was filtered through a nylon gauze, and then the fluid was centrifuged for 10 minutes $(300 \mathrm{~g})$. BALF supernatants were frozen at $-70^{\circ} \mathrm{C}$ and preserved until further processing. Cell pellets were suspended in $300 \mathrm{~mL}$ of phosphate-buffered saline (PBS) and gently spread on slides. The total cell count was measured in a Bürker chamber. Differential cell count was determined on two May-Grunwald-Giemsastained slides with the use of light microscopy. Of the rest of the cell pellets the smears were prepared for further analysis by immunofluorescence staining. The slides were air dried, frozen immediately, and stored at $-20^{\circ} \mathrm{C}$.

Five millilitres of blood from each patient was collected at the same time of the day. Serum was collected and stored at $-70^{\circ} \mathrm{C}$ for interleukin measurements.

\section{Immunofluorescence staining analysis}

Immunofluorescence reactions were performed on previously frozen BALF smears using a reaction in which the specific antibody-antigen complex is detected by secondary antibodies linked to a fluorescent marker.

Firstly, the slides were stained with mouse anti-CD163 monoclonal antibody (1 : 200, ab-100909, Abcam, USA). After 24 hours of incubation, the excess of unbound primary antibody was washed off with primary buffered saline solution of PBS. Next, it was incubation with rabbit anti-CCR7 monoclonal antibody (1 : 800 Abcam, USA, $24 \mathrm{~h}$ ). The primary antibodies were detected by secondary donkey anti-mouse polyclonal antibody with Alexa Fluor® 488 and donkey anti-rabbit polyclonal antibodies with Alexa Fluor ${ }^{\circledR} 555$ (1 : 200, Thermo Fisher Scientific, USA.). The sections were mounted using Vectashield with DAPI (4'6-diamidino-2-phenylindole, Vector Laboratories, USA). Immunofluorescence was analysed under an Eclipse 80i microscope (Nikon, Japan).

\section{Counting of macrophages in immunofluorescence staining}

The analysis in fluorescent microscopy consisted of the following steps: in five areas (200' magnification) all macrophages were counted (with any reaction) by two pathologists, independently. The result obtained from these five areas of view (number of cells) was assessed as representative for $100 \%$ of all macrophages. Next, macrophages that showed a positive reaction for: CCR7, CD163, or both CCR7 and CD163 in one cell were counted (Fig. 1).

Of the double-stained macrophages the following subtypes were defined:

CCR7+ CD163+,

CCR7 $7_{\text {low }} \mathrm{CD} 163+-$ with a weak reaction for CCR7 antigen and a strong reaction for CD163 antigen,

CCR7+CD163 $3_{\text {low }}$ - with a strong reaction for CCR7 antigen and a weak reaction for CD163.

\section{Enzyme-linked Immunosorbent Assay (ELISA)}

TGF- $\beta 1$ and IL- 10 concentrations were measured in the BALF supernatants and serum using a commercially available kit: Quantikine ELISA Human TGF- $\beta 1$ Immunoassay (R\&D System, USA) and Quantikine ELISA Human IL-10 Immunoassay (R\&D System, USA) according to the producer's recommendations. The absorbance was measured at $450 \mathrm{~nm}$ using a Microplate reader (model StatFox-2100; Awareness Technology, INC). The test sensitivity for TGF- $\beta 1$ was $4.61 \mathrm{pg} / \mathrm{ml}$. The lower limit of detection for IL-10 was $3.9 \mathrm{pg} / \mathrm{ml}$.

\section{Statistical analysis}

Statistica 12.0 software (StatSoft) was used for statistical analysis. For group comparison, the Mann-Whitney test and Kruskal-Wallis tests were used. Relations between the quantitative variables were analysed by Spearman correlations. A $p<0.05$ was considered as statistically significant.

\section{Results}

The characteristics of the investigated group and the results of BALF analysis were summarised in Table 1 . The men were significantly more numerous compared to the women, and the male patients were significantly younger than the female ones $(p<0.05)$. The highest incidence was for squamous cell carcinoma $(50.0 \%)$. Most patients were in a non-advanced stage of lung cancer. Due to the small number of patients in each group, we did not perform a comparison between groups with different types of cancer or between different stages of the disease. The macrophage proportion and count were similar when the clBALF and the hlBALF were compared. Also, the total cell count and the proportion of lymphocytes, neutrophils, and eosinophils were similar when the clBALF and the 


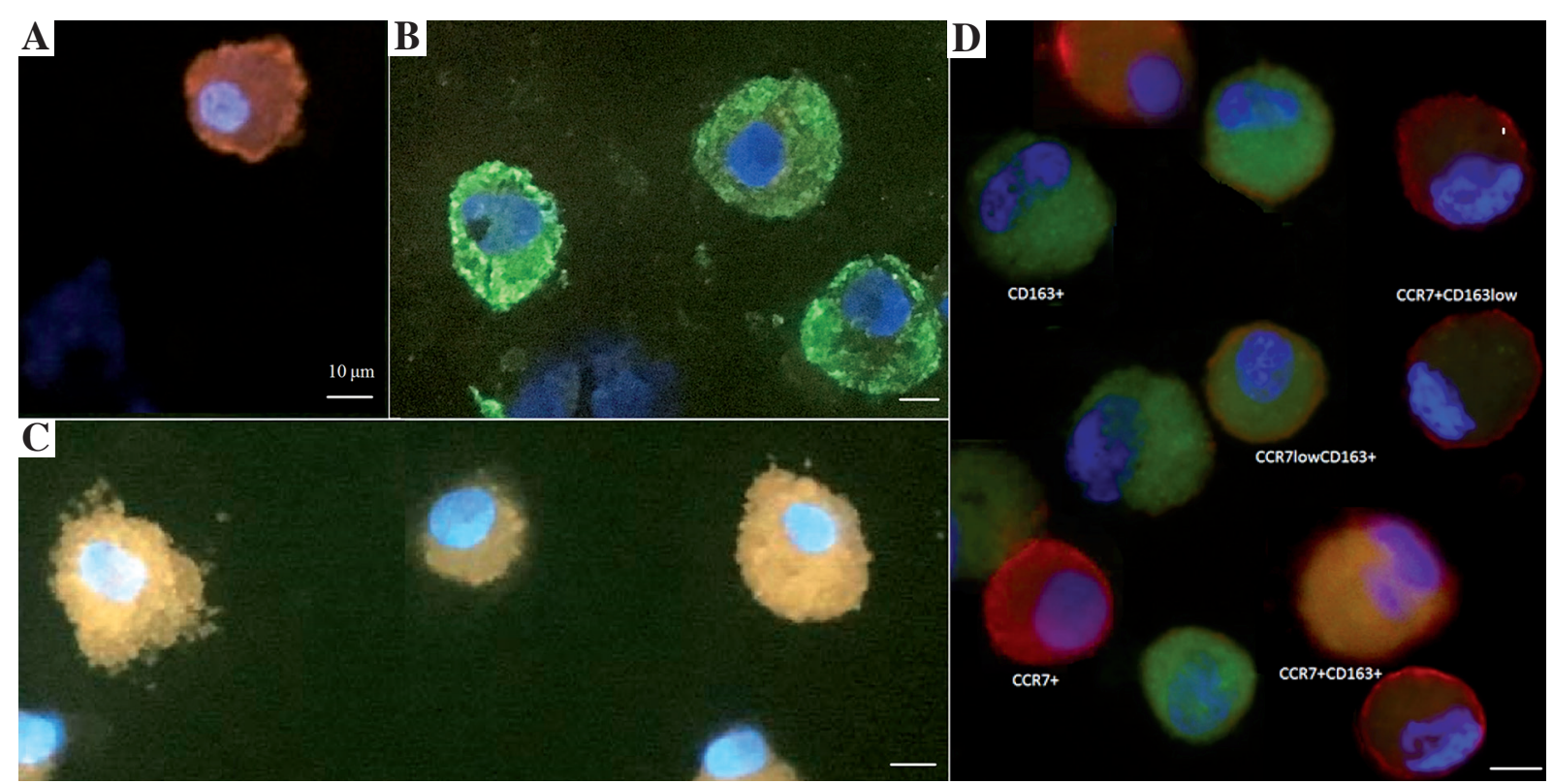

Fig. 1. Result of immunofluorescence reaction for CCR7 and CD163 markers: A) macrophages with a positive reaction for one marker: CCR7+ macrophages, B) macrophages with a positive reaction for one marker: CD163+ macrophages, C) double-stained macrophages CCR7+CD163+, D) example of all reactions for one patient. A microscope photo, scale bars: $10 \mu \mathrm{m}$. Immunofluorescence examination was performed in all 36 samples: 18 of the BALF from lung with cancer and 18 of the BALF from 'healthy' lung. The image of macrophages with reaction to CCR7 and CD163 was comparable in each slide. Here we present the examples of these reactions

hlBALF were compared. We noticed a higher proportion of CCR7+CD163+ macrophages, CCR7 ${ }_{\text {low }}$ CD163 + and tendency towards a higher proportion of CD163 + macrophages in the clBALF compared to the hlBALF. In addition, a lower proportion of CCR7+CD163 $3_{\text {low }}$ and tendency towards a lower proportion of CCR7+ macrophages were detected in the clBALF compared to the hIBALF. The differences are shown in Table 2 and Figure 2.

The median proportion of TGF- $\beta 1$ concentration in the clBALF supernatant was $15.6 \mathrm{pg} / \mathrm{ml}$ (1.1-48.4), which was lower than in the hlBALF supernatant: $33.8 \mathrm{pg} / \mathrm{ml}$ (4.7$62.9)$, difference non-significant. The median proportion of TGF- $\beta 1$ in the serum was lower than in the clBALF (not significant) and in the hlBALF (difference significant, $p<0.05)$, and the level was $10.0 \mathrm{pg} / \mathrm{ml}(4.1-14.3)$.

There was a positive significant correlation between the proportion of CD163+ macrophages and TGF- $\beta 1$ concentration in the clBALF $(r=0.6, p<0.05)$ but not in the hlBALF. In the hIBALF we observed a positive significant correlation between the proportion of CCR7+ macrophages and the TGF- $\beta 1$ concentration $(r=0.6, p<0.05)$ and between TGF- $\beta 1$ concentration and proportion of CCR7+CD163 $3_{\text {low }}$ cells $(r=0.6, p<0.05)$. This observation should be interpreted with caution because the proportion of cells is low.

We observed a significant negative correlation between the proportion of CCR7+CD163+ macrophages and TGF- $\beta 1$ concentration only in the $\operatorname{clBALF}(r=-0.7, p<0.05)$. We did not observe this correlation in the hlBALF.

The IL-10 concentration in the BALF was below the lower limit of detection.

\section{Discussion}

The present study shows a higher proportion of CD163+ and CCR7 ${ }_{\text {low }}$ CD163+ in the BALF from the direct tumour environment compared to the BALF from the 'healthy lung'. There was a greater proportion of CCR7+ and CCR7+CD163 $3_{\text {low }}$ macrophages in the 'healthy lung' BALF compared to the BALF from the lung cancer site. These results imply that the dominant population in the immediate vicinity of the tumour may be M2 macrophages, whereas in the 'healthy lung', macrophages similar to M1 are the dominant subpopulation. We used for the first time macrophages harvested by BAL to explore a possible role of these cells in a local immune response in malignancy. Due to the possibility of double staining, the immunofluorescence method proved valuable to show the direction of macrophage polarization.

Other authors also pointed at the presence of M2 macrophages in the tumour milieu. However, they focused on the presence of tumour-associated macrophages (TAM) in the tissue of resected tumours. Other authors presented M2 macrophages and their role as a negative prognostic 
factor for patients with lung cancer. Zhang et al. [35] assessed cancer-associated macrophages in adenocarcinoma by immunofluorescence. M1 macrophages were defined as CD68+iNOS+ cells, while M2 macrophages were CD68+CD206+. They showed that polarization in the direction of M2 phenotype predominates in cancer and is associated with a worse prognosis, possibly due to accelerated lymphangiogenesis and lymph node metastasis.

Ma et al. [36] evaluated cancer-related macrophages to determine whether the presence of a particular lung macrophage phenotype was related to the survival time of a patient with NSCLC. M1 macrophages were defined by the presence of CD68+HLA-DR+, and M2 macrophages by CD68+CD163+ in double immunohistochemical staining. These authors demonstrated polarization for M2 macrophages in approximately $70 \%$ of cases. There was no correlation between M2 macrophage density and survival time, but macrophage M1 phenotype was shown to be an independent positive predictor for patient survival. In another study, Carus et al. [37] assessed by immunohistochemistry the presence of M2 macrophages defined as CD163+ in NSCLC tumour sections. It was shown that a higher CD163+ macrophage density correlated with a CRP level and lymph node metastasis, but no association was found with survival time.

Chen et al. [38] analysed BALF M2 macrophages by immunocytochemical staining in patients with squamous cell carcinoma, adenocarcinoma, and small cell carcinoma, in comparison to BALF macrophages from patients with non-specific chronic pneumonia and from healthy individuals. The study demonstrated that CD163+ macrophages are a dominant subpopulation in the BALF of cancer patients with the highest percentage in squamous cell carcinoma. Our results are consistent with these results (although, due to the limited number of patients, the macrophage subpopulations were not analysed for the histological type of lung cancer).

$\mathrm{Yu}$ et al. presented an interesting observation in terms of methodology [39]. They evaluated a macrophage population in BALF and in lung tissue using multicolour cytometry and confocal microscopy. They showed that all tested macrophages were positive for CD206+ antigen, whereas the expression of CD163+ antigen on these cells varied depending on the location in the lung. Macrophages CD163+ were found in the alveolar space, but CD163- macrophages were closely related to the lung interstitium and were not detected in the BALF. This observation justifies our choice of the CD163+ marker to identify the M2 population in BALF, while the choice of the CD206 marker appears to be questionable. Yuan et al. [40] evaluated the polarization of macrophages in NSCLC using flow cytometry, gene profile, and expression of cytokines. It has been shown that CCR7, CD23, and CD163 are specific surface markers for M1, M2a, and M2c phenotypes, respectively, and additionally the CD206 marker is common to both M2 subtypes.
Table 1. Characteristic of the study population

\begin{tabular}{|c|c|c|}
\hline Characteristic & \multicolumn{2}{|c|}{ Patients } \\
\hline Sex F/M (n) & \multicolumn{2}{|c|}{$6 / 12$} \\
\hline Age (mean \pm SD years) & \multicolumn{2}{|c|}{$68.4 \pm 7.3$} \\
\hline Women (mean \pm SD years) & \multicolumn{2}{|c|}{$72.3 \pm 7.3$} \\
\hline Men (mean \pm SD years) & \multicolumn{2}{|c|}{$66.4 \pm 6.7$} \\
\hline \multicolumn{3}{|l|}{ Smoking history, $n(\%)$} \\
\hline Smokers & \multicolumn{2}{|c|}{$6(33.3)$} \\
\hline Ex-smokers & \multicolumn{2}{|c|}{$8(44.4)$} \\
\hline Never-smokers & \multicolumn{2}{|c|}{$4(22.2)$} \\
\hline \multicolumn{3}{|l|}{ Histology, \% } \\
\hline Squamous cell carcinoma & \multicolumn{2}{|c|}{50.0} \\
\hline Adenocarcinoma & \multicolumn{2}{|c|}{22.2} \\
\hline Not otherwise specified & \multicolumn{2}{|c|}{22.2} \\
\hline \multicolumn{3}{|l|}{ Stage of disease $(n, \%)$} \\
\hline I & \multicolumn{2}{|c|}{$4(22.2)$} \\
\hline II & \multicolumn{2}{|c|}{$11(61.1)$} \\
\hline IIIA & \multicolumn{2}{|c|}{$3(16.7)$} \\
\hline BALF description & clBALF & hlBALF \\
\hline Total cell count $\left(\times 10^{6}\right)$ & $7.1 \pm 3.4$ & $5.2 \pm 2.7$ \\
\hline \multicolumn{3}{|l|}{ MGG staining (mean $\pm \mathrm{OD}$ ) } \\
\hline Macrophages (\%) & $49.5 \pm 24.6$ & $54.1 \pm 23.2$ \\
\hline Lymphocytes (\%) & $28.4 \pm 14.3$ & $22.2 \pm 10.2$ \\
\hline Neutrophils (\%) & $22.5 \pm 21.7$ & \pm 22.5 \\
\hline Eosinophils (\%) & 0 & 0 \\
\hline
\end{tabular}

$\overline{B A L F-\text { bronchoalveolar lavage fluid, } c l B A L F-\text { bronchoalveolar lavage fluid }}$ from the lung affected by cancer, hlBALF - bronchoalveolar lavage fluid from the healthy lung, $F$-female, $M$-male, MGG-May-Grunwald Giemsa staining, NOS - not otherwise specified

The study demonstrated that M2a and M2c are involved in the promotion of lung cancer invasion and tumour growth. In contrast, M1 suppresses angiogenesis and increases cell sensitivity to cisplatin, decreases the viability of tumour cells, and induces aging and apoptosis of these cells. M1 macrophages significantly correlated with the longer overall survival. These results confirm that macrophage phenotyping can be used as a prognostic indicator in patients with lung cancer and may be the target of immunomodulation therapy in the future.

The results of the current study indicate that M1 and M2 macrophages may contain both CCR7 and CD163 antigens, indicating that polarization of macrophages is not always one-way and these cells are highly plastic. It is possible that the subpopulations penetrate among themselves, perhaps depending on the stage of the disease and the character of local immune response, and it is an individual process. The results indicate that there is no clear distinction between the phenotype M1 and M2, and these 
Table 2. Differences in the proportion of macrophage subpopulations depending on the distance from the tumour, on the basis of bronchoalveolar lavage fluid (BAL) analysis from the lung affected by cancer (clBALF) and from the healthy symmetrical lung (hlBALF) as a control from the same patient

\begin{tabular}{|c|c|c|c|c|}
\hline & Parameter & clBALF & hIBALF & $p$ \\
\hline & Macrophages (\%) & Median (p25-p75) & Median (p25-p75) & \\
\hline 1 & CCR7+ & $0.7(0.0-2.6)$ & $6.0(0.8-18.2)$ & - \\
\hline 2 & CD163+ & $12.4(7.7-23.6)$ & $7.7(2.4-32.5)$ & - \\
\hline 3 & CCR7+CD163+ & $84.6(64.7-90.6)$ & $51.9(34.9-83.3)$ & $0.0496^{*}$ \\
\hline 4 & $\mathrm{CCR}_{\text {low }} \mathrm{CD} 163+$ & $92.9(84.0-97.4)$ & $56.0(48.0-70.6)$ & $0.0194^{*}$ \\
\hline 5 & CCR7+CD163 ${ }_{\text {low }}$ & $7.8(0.0-12.1)$ & $40.2(18.7-56.8)$ & $0.0007^{*}$ \\
\hline
\end{tabular}

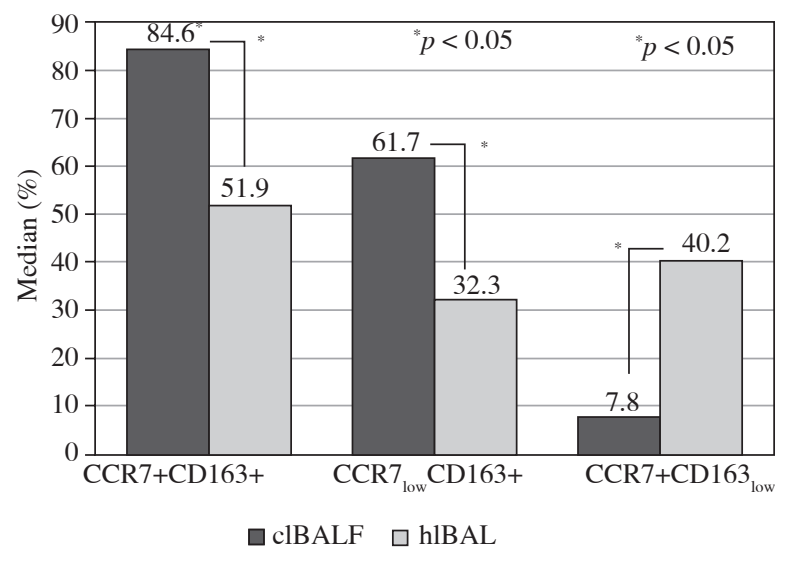

Fig. 2. Median proportion of macrophage subpopulations in bronchoalveolar lavage fluid (BALF) of lung cancer patients from the lung affected by cancer (clBALF) and from the healthy symmetrical lung (hlBALF). The proportion of double stained cells: CCR7+CD163+ as a proportion of all macrophages is presented (first column). The subpopulations CCR $7_{\text {low }}+\mathrm{CD} 163+$ and CCR7+CD163 $3_{\text {low }}$ are presented as a proportion of CCR7+CD163 cells (second and third column). ( $\left.{ }^{*} p<0.05\right)$. Data are presented as median values

cells, depending on the immunological status of the patient and the cytokine profile in the tumour environment, are in the stage between the M1 and M2 phenotypes [18].

TGF- $\beta 1$ concentration was higher in the BALF than in the serum, and in the "healthy" BALF it was the highest. Chen et al. [41] evaluated TGF- $\beta$ in the BALF of patients with lung cancer in relation to patients with non-neoplastic diseases (pneumonia, tuberculosis, or sarcoidosis). Patients with lung cancer had a higher TGF- $\beta$ level compared to the control groups. TGF- $\beta$ concentration in the BALF of non-neoplastic patients was comparable to the serum TGF- $\beta$ concentration in the lung cancer pa- tients in the present work ( $8.4 \mathrm{vs} .10 \mathrm{pg} / \mathrm{ml}$, respectively). Domagała-Kulawik et al. [42] compared TGF- $\beta$ levels in the BALF from patients with lung cancer with healthy volunteers and found a significantly higher TGF- $\beta$ concentration in those with lung cancer than in the healthy ones. The TGF- $\beta$ level in the lung cancer BALF grew with the increase of disease progression. Also, in their study, the TGF- $\beta$ concentration in the BALF from the healthy subjects was comparable to the serum TGF- $\beta$ level in the lung cancer patients in the present study (10.1 vs. $10.0 \mathrm{pg} / \mathrm{ml}$, respectively). The above observations imply that measuring the TGF- $\beta$ concentration in the direct tumour environment may be more important than assessing this cytokine in serum. In our study, a correlation between TGF- $\beta 1$ concentration and macrophage populations was investigated. We found a positive correlation of TGF- $\beta 1$ concentration with the proportion of CD163+ cells in the tumour environment. In contrast, in the BALF from the "healthy lung" we showed a positive correlation between TGF- $\beta 1$ concentration and the proportion of CCR7+ cells. A negative correlation between the proportion of double-stained CCR7+CD163+ cells and TGF- $\beta 1$ concentration in BALF was observed only in the tumour environment. Our results are consistent with the observation of the multifunctional role of TGF- $\beta$ [43, 44]. Mia et al. [45] elaborated a protocol for the polarization and production of specific immunosuppressive human blood monocytes. This work has resulted in optimal and effective cytokine dosing of IL-4, IL-10, and TGF- $\beta$ for in vitro induction of immunosuppressive human M2 macrophages, characterised by a subsequent expression of surface receptor, cytokine profile, function, and ability to suppress cytotoxic T-cell proliferation by these cells. IL-4, IL-10, and TGF- $\beta$-cytokine combinations have been shown to strongly stimulate M2 macrophage generation, while at the same time having a strong deactivating effect on M1 macrophages.

Our results and the above-presented data imply that the TGF- $\beta$ function is strongly related to the nature of the en- 
vironment in which it is found and confirm the high plasticity of this cytokine. On one hand, TGF- $\beta 1$ in the "healthy lung' environment correlates with M1 with proinflammatory properties; on the other hand, in the tumour site it correlates with M2 with immunosuppressive properties.

The weakness of this study is the small number of patients and the lack of a possibility to subgroup them according to histology and stage of the disease. Nonetheless, the results are the first and mark a direction for further research.

\section{Conclusions}

The above results confirmed the differences between the two compartments: the lung with tumour and the healthy lung and the usefulness of BALF analysis in the evaluation of the nature of local immune response. The usefulness of the immunofluorescence method with CCR7 and CD163 markers in the evaluation of BALF macrophage polarization in the lung cancer microenvironment was presented with a predominance of immunosuppressive macrophages (M2) and a place of TGF- $\beta 1$ in this process. In the era of searching for biomarkers for lung cancer immunotherapy our study brings a new aspect.

\section{Acknowledgements}

The authors would like to thank Malgorzata Proboszcz, PhD student, for assistance with the ELISA test analysis.

This study was supported by grants from: Medical University of Warsaw: 1WU/PM11D/14 and Military Institute of Medicine: 13/W/2018 488.

The authors declare no conflict of interest.

\section{References}

1. Dasanu CA, Sethi N, Ahmed N (2012): Immune alterations and emerging immunotherapeutic approaches in lung cancer. Expert Opin Biol Ther 12: 923-937.

2. Domagala-Kulawik J, Osinska I, Hoser G (2014): Mechanisms of immune response regulation in lung cancer. Transl Lung Cancer Res 3: 15-22.

3. Pardoll DM (2012): The blockade of immune checkpoints in cancer immunotherapy. Nat Rev Cancer 12: 252-264.

4. Gordon S, Taylor PR (2005): Monocyte and macrophage heterogeneity. Nat Rev Immunol 5: 953-964.

5. Arora S, Dev K, Agarwal B, et al. (2018): Macrophages: Their role, activation and polarization in pulmonary diseases. Immunobiology 223: 383-396.

6. Martinez FO, Sica A, Mantovani A, Locati M (2008): Macrophage activation and polarization. Front Biosci 13: 453-461.

7. Sica A, Larghi P, Mancino A, et al. (2008): Macrophage polarization in tumour progression. Semin Cancer Biol 18: 349-355.
8. Comito G, Giannoni E, Segura CP, et al. (2014): Cancer-associated fibroblasts and M2-polarized macrophages synergize during prostate carcinoma progression. Oncogene 33: 2423-2431.

9. Harvey NL, Gordon EJ (2012): Deciphering the roles of macrophages in developmental and inflammation stimulated lymphangiogenesis. Vasc Cell 4: 15.

10. Nazimek K, Bryniarski K (2012): [The biological activity of macrophages in health and disease]. Postepy Hig Med Dosw (Online) 66: 507-520.

11. Biswas SK, Mantovani A (2010): Macrophage plasticity and interaction with lymphocyte subsets: cancer as a paradigm. Nat Immunol 11: 889-896.

12. Murray PJ, Allen JE, Biswas SK, et al. (2014): Macrophage activation and polarization: nomenclature and experimental guidelines. Immunity 41: 14-20.

13. Mantovani A, Sozzani S, Locati M, et al. (2002): Macrophage polarization: tumor-associated macrophages as a paradigm for polarized M2 mononuclear phagocytes. Trends Immunol 23: 549-555.

14. Aron-Wisnewsky J, Tordjman J, Poitou C, et al. (2009): Human adipose tissue macrophages: $\mathrm{m} 1$ and $\mathrm{m} 2$ cell surface markers in subcutaneous and omental depots and after weight loss. J Clin Endocrinol Metab 94: 4619-4623.

15. Mantovani A, Sica A (2010): Macrophages, innate immunity and cancer: balance, tolerance, and diversity. Curr Opin Immunol 22: 231-237.

16. Imaizumi K, Kawabe T, Ichiyama S, et al. (1999): Enhancement of tumoricidal activity of alveolar macrophages via CD40-CD40 ligand interaction. Am J Physiol 277 (1 Pt 1): L49-57.

17. Zeyda M, Farmer D, Todoric J, et al. (2007): Human adipose tissue macrophages are of an anti-inflammatory phenotype but capable of excessive pro-inflammatory mediator production. Int J Obes (Lond) 31: 1420-1428.

18. Osinska I, Wolosz D, Domagala-Kulawik J (2014): Association between M1 and M2 macrophages in bronchoalveolar lavage fluid and tobacco smoking in patients with sarcoidosis. Pol Arch Med Wewn 124: 359-364.

19. Bono MR, Elgueta R, Sauma D, et al. (2007): The essential role of chemokines in the selective regulation of lymphocyte homing. Cytokine Growth Factor Rev 18: 33-43.

20. Woodland DL, Kohlmeier JE (2009): Migration, maintenance and recall of memory $\mathrm{T}$ cells in peripheral tissues. Nat Rev Immunol 9: 153-161.

21. Tateyama M, Fujihara K, Misu T, Itoyama Y (2009): CCR7+ myeloid dendritic cells together with CCR7+ T cells and CCR7+ macrophages invade CCL19+ nonnecrotic muscle fibers in inclusion body myositis. J Neurol Sci 279: 47-52.

22. Badylak SF, Valentin JE, Ravindra AK, et al. (2008): Macrophage phenotype as a determinant of biologic scaffold remodeling. Tissue Eng Part A 14: 1835-1842.

23. Oh J, Riek AE, Weng S, et al. (2012): Endoplasmic reticulum stress controls M2 macrophage differentiation and foam cell formation. J Biol Chem 287: 11629-11641.

24. Wojtan P, Mierzejewski M, Osinska I, Domagala-Kulawik J (2016): Macrophage polarization in interstitial lung diseases. Cent Eur J Immunol 41: 159-164.

25. Wrzesinski SH, Wan YY, Flavell RA (2007): Transforming growth factor-beta and the immune response: implications for anticancer therapy. Clin Cancer Res 13: 5262-5270.

26. Li MO, Wan YY, Sanjabi S, et al. (2006): Transforming growth factor-beta regulation of immune responses. Annu Rev Immunol 24: 99-146. 
27. Gong D, Shi W, Yi SJ, et al. (2012): TGF-beta signaling plays a critical role in promoting alternative macrophage activation. BMC Immunol 13: 31.

28. Moore KW, de Waal Malefyt R, Coffman RL, O'Garra A (2001): Interleukin-10 and the interleukin-10 receptor. Annu Rev Immunol 19: 683-765.

29. Mantovani A (2006): Macrophage diversity and polarization: in vivo veritas. Blood 108: 408-409.

30. Osinska I, Domagala-Kulawik J (2013): [Bronchoalveolar lavage in lung cancer - diagnostic value and assessment of the anti-cancer immune response]. Postepy Hig Med Dosw (Online) 67: 1119-1127.

31. Osinska I, Stelmaszczyk-Emmel A, Polubiec-Kownacka M, et al. (2016): CD4+/CD25(high)/FoxP3+/CD127- regulatory $\mathrm{T}$ cells in bronchoalveolar lavage fluid of lung cancer patients. Hum Immunol 77: 912-915.

32. Kwiecień I, Stelmaszczyk-Emmel A, Polubiec-Kownacka M, et al. (2017): Elevated regulatory T cells, surface and intracellular CTLA-4 expression and interleukin-17 in the lung cancer microenvironment in humans. Cancer Immunol Immunother 66: 161-170.

33. Detterbeck FC, Postmus PE, Tanoue LT (2013): The stage classification of lung cancer: Diagnosis and management of lung cancer, $3^{\text {rd }}$ ed: American College of Chest Physicians evidence-based clinical practice guidelines. Chest 143 (5 Suppl): e191S-210S.

34. Chcialowski A, Chorostowska-Wynimko J, Fal A, et al. (2011): Recommendation of the Polish Respiratory Society for bronchoalveolar lavage (BAL) sampling, processing and analysis methods. Pneumonol Alergol Pol 79: 75-89.

35. Zhang B, Yao G, Zhang Y, et al. (2011): M2-polarized tumor-associated macrophages are associated with poor prognoses resulting from accelerated lymphangiogenesis in lung adenocarcinoma. Clinics (Sao Paulo) 66: 1879-1886.

36. Ma J, Liu L, Che G, et al. (2010): The M1 form of tumorassociated macrophages in non-small cell lung cancer is positively associated with survival time. BMC Cancer 10: 112.

37. Carus A, Ladekarl M, Hager H, et al. (2013): Tumor-associated neutrophils and macrophages in non-small cell lung cancer: no immediate impact on patient outcome. Lung Cancer 81: 130-137.

38. Chen L, Li Q, Zhou XD, et al. (2014): Increased pro-angiogenic factors, infiltrating neutrophils and CD163(+) macrophages in bronchoalveolar lavage fluid from lung cancer patients. Int Immunopharmacol 20: 74-80.

39. Yu YR, Hotten DF, Malakhau Y, et al. (2016): Flow Cytometric Analysis of Myeloid Cells in Human Blood, Bronchoalveolar Lavage, and Lung Tissues. Am J Respir Cell Mol Biol 54: 13-24.

40. Yuan A, Hsiao YJ, Chen HY, et al. (2015): Opposite Effects of M1 and M2 Macrophage Subtypes on Lung Cancer Progression. Sci Rep 5: 14273.

41. Chen Z, Xu Z, Sun S, et al. (2014): TGF-beta1, IL-6, and TNF-alpha in bronchoalveolar lavage fluid: useful markers for lung cancer? Sci Rep 4: 5595.

42. Domagala-Kulawik J, Hoser G, Safianowska A, et al. (2006): Elevated TGF-beta1 concentration in bronchoalveolar lavage fluid from patients with primary lung cancer. Arch Immunol Ther Exp (Warsz) 54: 143-147.

43. Wahl SM (1994): Transforming growth factor beta: the good, the bad, and the ugly. J Exp Med 180: 1587-1590.
44. Wahl SM, Orenstein JM, Chen W (2000): TGF-beta influences the life and death decisions of T lymphocytes. Cytokine Growth Factor Rev 11: 71-79.

45. Mia S, Warnecke A, Zhang XM, et al. (2014): An optimized protocol for human M2 macrophages using M-CSF and IL-4/ IL-10/TGF-beta yields a dominant immunosuppressive phenotype. Scand J Immunol 79: 305-314. 